\title{
STEM and TEM: Disparate Magnification Definitions and a Way Out.
}

\author{
E. Voelk1 ${ }^{1}$, David Hoyle ${ }^{2}$, Jane Howe ${ }^{1}$, H. Inada ${ }^{3}$ and T. Yotsuji ${ }^{3}$ \\ 1. Hitachi High Technologies America, Clarksburg, MD 20871 \\ 2. Hitachi High-Technology, Ltd., 89 Rexdale Blvd., Toronto, M9W 6A4 Canada \\ 3. Science \& Medical Systems Business Group, Hitachi High-Technologies Corp., Hitachinaka, Ibaraki \\ 312-8504 Japan.
}

Magnification ranges for STEM and TEM cover a wide range. For example, in [1], the STEM magnification is rated as $\times 200$ to $\times 150 \mathrm{M}$, while the TEM magnification is rated as $\times 20$ to $\times 2 \mathrm{M}$. As a comparison, the STEM magnification range for the recently introduced Hitachi HF5000 is x20 to x4M, while for TEM the range is rated from $\mathrm{x} 100$ to $\mathrm{x} 1.5 \mathrm{M}$. Such major discrepancies in STEM magnification definitions between vendors make comparison of such instruments unnecessarily difficult.

Historically, magnification for the TEM mode was uniquely defined as the magnification (mag) between the plane of the sample and the plane of the photographic film. The introduction of digital cameras caused problems insofar as they are typically positioned in different planes, both above or below the reference-plane of the film: in some cases the camera may be looking at the viewing screen or be located at the end of a Gatan imaging filter.

Historically, magnification for the STEM mode was defined via a Polaroid camera, meaning a photo off the surface of a scintillator tube. However, with digital STEM imaging a clear definition for STEM magnification seems to be missing and a definition via the computer monitor or projector appears vague.

To be short and to the point, we believe reproducibility is one of the most important virtues of science. Therefore, a given magnification should describe the setting of the microscope in terms of its optics. Each electron microscope has, for a given magnification, a default lens setting and knowing the magnification therefore should allow reproduction of that setting. The actual position of the camera does not describe the optics of the microscope and should not play a role; even more so, as there are competing cameras with different pixel sizes and numbers of pixels for the same spot on the microscope. Therefore, a well-defined image reference-plane below the last projector lens was selected. But now the STEM mode needs to be defined in a similar manner.

The STEM mode has a unique feature: for a given magnification, the (full) FOV (field of view) remains the same, independent of the number of pixels used for creating the STEM image (imaging sub-areas should not change the magnification definition but rather be handled as a software detail stored with the image). Under these conditions, it seems appropriate to connect the STEM magnification with its FOV. Thus it only remains to be determined how the STEM field of view relates to the TEM field of view.

The most convenient behaviour of the microscope, when switching from TEM to STEM or vice versa, is to maintain (at least approximately) sample position and FOV. This can be implemented by defining a FOV for the (TEM) camera in the image reference-plane.

Hitachi defined the image reference-plane for the magnification to coincide with a $1024 \times 1024$ pixel camera with a pixel size of $21.97 \mu \mathrm{m}$. Thus at a magnification of $\mathrm{x} 1.5 \mathrm{M}$, one pixel corresponds to 
$14.6 \mathrm{pm}$ in the object plane and a FOV of $15 \times 15 \mathrm{~nm}^{2}$. For an assumed maximum resolution of $0.6 \AA$ in TEM, this would allow 4 pixels per smallest resolvable detail; an easily sufficient magnification for allowing a good MTF (modulation transfer function) given the relatively large pixel size of $21.97 \mu \mathrm{m}$. For STEM, the maximum magnification of the HF5000 is $\mathrm{x} 4 \mathrm{M}$, resulting in a FOV of $5.625 \times$ $5.625 \mathrm{~nm}^{2}$. Selecting an acquisition mode of $4096 \times 4096$ pixels, each pixel of the STEM image now has a size of $1.37 \times 1.37 \mathrm{pm}^{2}$. For a maximum resolution of $0.6 \AA$ in STEM, this corresponds to an oversampling of more than 20 times (in alignment with the Nyquist sampling theorem; 2 pixels per smallest detail).

An example where such an approach has already been implemented is the Hitachi HF5000. In Figure 1, a comparison of a TEM image and a STEM image acquired at the same magnification is shown. The field of view is very close, as should be.

Although this may be puzzling at first, we believe that TEM and STEM magnification should be aligned via the FOV (field of view). We further believe that the maximum needed STEM magnification is directly connected with pixel size in the object plane. And we believe that a magnification yielding effectively a $1 \times 1 \mathrm{pm}^{2}$ pixel size is more than sufficient in general (whether its 'labelled' $\mathrm{x} 4 \mathrm{M}$ or $\mathrm{x} 150 \mathrm{M}$ ).

\section{References:}

[1] http://www.jeol.de/electronoptics-en/products/electron-and-ion-optics/transmission-electronmicroscopes/200-kv-tem-feg-tem/jem-f200.php
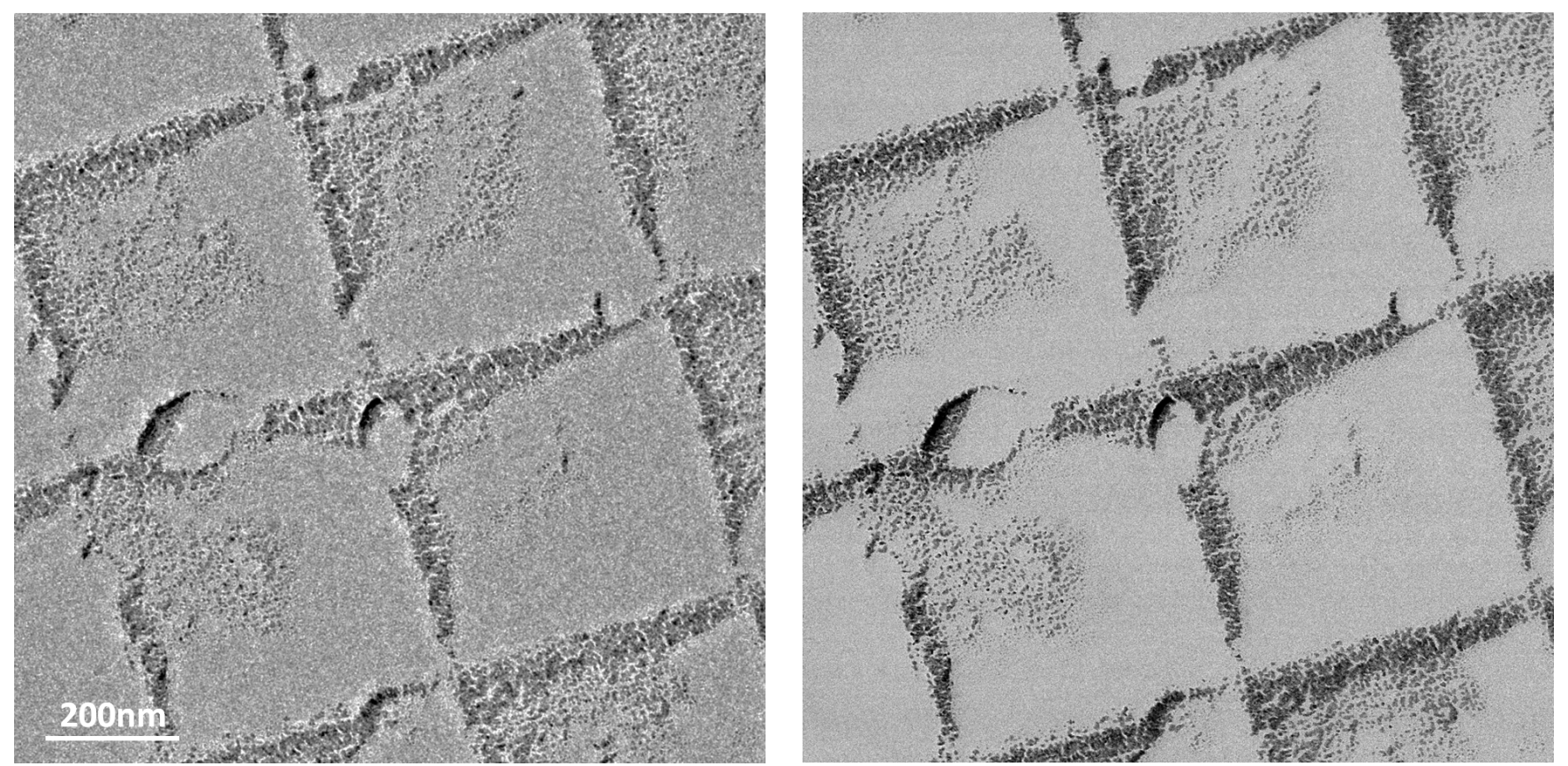

Figure 1. Comparison of a TEM image (left) and a STEM image (right) at a magnification of x20,000. Both images show the same field of view. Such a convenience feature is possible with the magnification definitions for TEM and STEM suggested here. 